\title{
Determination of the Calibration Factor of Superconducting Gravimeter 057 at the Lhasa Station: A frequency-Domain Approach
}

\author{
Xiaodong Chen *, Heping Sun, Houze Xu, Jianqiao Xu, Xinghua Hao, and Ming Liu \\ State Key Laboratory of Geodesy and Earth's Dynamics, Institute of Geodesy and Geophysics, \\ Chinese Academy of Sciences, Wuhan, China
}

Received 4 March 2012, accepted 3 December 2012

\begin{abstract}
In this paper, the calibration factor of the superconducting gravimeter 057 (SG057) at the Lhasa station is accurately determined for the first time with a frequency-domain approach and the data recorded by the LaCoste Romberg Earth Tides No. 20 (LCR-ET20) gravimeter which is installed nearby SG057. The advantage of the frequency-domain approach is that it eliminates the influence of different gravimeter drifts on the determination of the calibration factor. The determined calibration factor of SG057 is $(-77.7358 \pm 0.0409) \times 10^{-8} \mathrm{~m} \mathrm{~s}^{-2} \mathrm{~V}^{-1}$, and the relative accuracy is about $0.5 \%$. The newly determined calibration factor has been calculated with the Wuhan international tidal gravity reference values (ITGRVs) based on the data recorded by LCR-ET20 at the Wuhan station before it was installed at the Lhasa station. A high-precision synthesized gravity tide is achieved at the Lhasa station with accurate tidal parameters and theoretical tidal parameters for the long tidal waves from the Dehant-Defraigne-Wahr (DDW) earth tide model. The synthesized gravity tide can be used for tidal gravity corrections of future gravity measurements in the Tibetan area. The linear gravimeter drift of SG057 is estimated by gravity records without the effects of land uplift, atmosphere and polar motion yielding a rate of $6.8 \times 10^{-8} \mathrm{~m} \mathrm{~s}^{-2} \mathrm{yr}^{-1}$.
\end{abstract}

Key words: Superconducting gravimeter 057, LCR-ET20 gravimeter, Calibration factor, Tidal amplitude, Tibetan area

Citation: Chen, X., H. Sun, H.Xu, J. Xu, X. Hao, and M. Liu, 2013: Determination of the calibration factor of superconducting gravimeter 057 at the Lhasa station: A frequency-domain approach. Terr. Atmos. Ocean. Sci., 24, 629-636, doi: 10.3319/TAO.2012.12.03.02(TibXS)

\section{INTRODUCTION}

Tibet is an active tectonic area of the earth. Its uplift is a scientific hotspot in the study of geosciences (Lu et al. 2004; Zhou et al. 2007; Yi et al. 2008; Xing et al. 2011). Several types of observations, such as the Global Positioning System (GPS), seismology, gravity, leveling, have been applied in this area for scientific studies. However, there is no long, precise and continuous tidal gravity observations until the superconducting gravimeter 057 (SG057) was installed in November, 2009 at the Lhasa station. Continuous gravity observations from SG057 are expected to show detailed behavior in the gravity changes caused by the uplift of the Tibetan Plateau and other factors (Hinderer and Crossley 2004; Crossley et al. 2005; Kroner and Weise 2011).

Before data recorded by a SG can be used for gravity analysis, the data must be calibrated with its calibration

\footnotetext{
* Corresponding author

E-mail: chenxd@whigg.ac.cn
}

factor. In a typical case, an apriori calibration factor is given by the manufacturer of a SG. For SG057, it is $-79.5 \times$ $10^{-8} \mathrm{~m} \mathrm{~s}^{-2} \mathrm{~V}^{-1}$. However, the given apriori calibration factor may be altered over time. Our earlier data investigation shows that the apriori calibration factor of SG057 is about $2 \%$ larger by comparing the observed tidal parameters from SG057 with those from the LaCoste Romberg Earth Tide No. 20 (LCR-ET20) gravimeter installed in an adjacent observing room. Therefore, the calibration factor of SG057 at Lhasa must be re-determined before use.

Under the hypothesis that tidal gravity variations recorded by different gravimeters are the same if these gravimeters are not far away distant from each other, the calibration factor of a gravimeter can be calculated by comparing its observation with the data recorded by the gravimeter with a known calibration factor. In most cases, the calibration factor of a SG is determined using a linear regression with the data recorded in parallel by an absolute gravimeter (Hinderer et al. 1991; Francis 1997; Sun et al. 2001; Meurers 
2002; Riccardi et al. 2012). We also collected observations by the absolute gravimeter FG5-214 in October 2010 simultaneously with the SG057. However, the calibration for the SG057 using FG5-214 data was unsuccessful because of the poor data quality of the FG5-214 in that campaign. Because of this, in this paper, we attempt to use the data observed with the LCR-ET20 to determine the calibration factor of the SG057. LCR-ET20 was installed in November 2009, which is near the time of installing SG057. These two gravimeters are separated by a few meters. Because there are different gravimeter drifts for the LCR-ET20 and SG057, calibrating the calibration factor of SG057 by LCR-ET20 in the frequency domain is preferred over the time domain (Meurers 2012). In the following sections, we will explain the frequency-domain calibration.

In addition to the calibration factor, the observation accuracy and the gravimeter drift of the SG057 can also be estimated. For a tidal gravimeter, the standard deviation from the harmonic analysis is considered as the observation accuracy. The gravimeter drift of an SG can be either exponential or linear. The exponential gravimeter drift primarily appears at the beginning of the installation. After at least one or several weeks, the gravimeter drift may gradually become linear. If the exponential drift does not occur at the beginning of the installation, the gravimeter drift could be assumed to be linear for the SG (Van Camp and Francis 2007).

In this paper, the frequency-domain approach is derived first. Then, the harmonic analysis results of LCR-ET20 and SG057 with the apriori calibration factors are presented. Based on the Wuhan international tidal gravity reference values (ITGRVs) (Xu et al. 2000) and the harmonic analysis results, the calibration factors of the LCR-ET20 and SG057 are calculated. Using the newly determined calibration factor of the SG057, high-precision tidal parameters are then computed. After the synthesized gravity tide and gravity residual are calculated, the gravimeter drift of SG057 is estimated.

\section{THE FREQUENCY-DOMAIN APPROACH OF CALIBRATION}

The frequency-domain approach of calibration in this paper is based on amplitude factors and their accuracies of the main tidal waves that are determined from the harmonic analysis. By the harmonic analysis, the signals observed by a gravimeter in time domain can be expressed by the amplitude factors and phase shifts in the frequency domain. Because the calculation is carried out in the frequency domain, this method eliminates the influence of different gravimeter drifts on the determination of the calibration factor which highlights the advantage of this method. It should be noted that the setting parameters (including wave groups, tidal potential catalogue, etc.) in the harmonic analysis for both two gravimeters (LCR-ET20 and SG057) must be the same. According to the magnitude of the amplitude, the amplitude factors and their accuracies of the main waves are selected for calculation of the calibration factor. For example, the $\mathrm{O} 1, \mathrm{~K} 1$ and $\mathrm{M} 2$ are selected in the calculation of the calibration factor; S1 and S2 are not selected because they are seriously affected by atmospheric effects, even though their amplitudes are relatively large enough.

The amplitude factors and their accuracies of the main waves selected for the gravimeter with a known calibration factor $C_{k n o w n}$ are denoted as $y_{k} \pm \Delta y_{k}, k=1,2, \ldots, n$, and those for the gravimeter with an apriori calibration factor $C_{\text {apriori }}$ are denoted as $x_{k} \pm \Delta x_{k}, k=1,2, \ldots, n$, where $n$ is the number of main waves selected. The theoretical amplitude and observed amplitude of the $k^{\text {th }}$ main wave are $A_{k}^{\text {theo }}$ and $A_{k}^{o b}$ respectively. Because the two gravimeters are separated by a few meters, $A_{k}^{o b}$ for these two gravimeters can be considered identical, we have

$A_{k}^{o b}=y_{k} \cdot A_{k}^{\text {theo }}=S_{k} \cdot x_{k} \cdot A_{k}^{\text {theo }}$

where $S_{k}$ is the ratio value of $y_{k}$ to $x_{k}$. If $C_{\text {known }}$ and $C_{\text {apriori }}$ are accurate enough, $S_{k}$ should be or nearly be 1.0. Equation (1) shows the linear relationship between actual calibration factor $C$ (to be determined) and $C_{\text {apriori }}$, but only at the frequency band of the $k^{\text {th }}$ main wave, i.e., we have $C_{k}=$ $S_{k} \cdot C_{\text {apriori }}$. In addition, it is assumed that the calibration factors at all tidal frequency bands are identical. Because $C_{\text {apriori }}$ is a constant, a mean $C$ value is determined from the $S_{k}$ values at the frequency band of the $k^{\text {th }}$ main wave. In addition, because the estimated tidal parameters with small-amplitude tidal waves contain relatively large errors, only tidal waves with large amplitude are selected to compute $C$.

The simple mean value of $S_{k}$ is denoted as $S$, and is

$S=\frac{1}{n} \sum_{k=1}^{n} S_{k}=\frac{1}{n} \sum_{k=1}^{n} \frac{y_{k}}{x_{k}}$

However, we did not use the simple mean of Eq. (2). Rather, we used the inverted accuracy as the data weight. Specifically, the ratio of the reciprocal of the accuracy $\Delta x_{k}$ to the sum of the reciprocals of the accuracies for the selected $n$ main waves is the weight of $S_{k}$. Let the weight of $S_{k}$ be $w_{k}$ and the total weight be $w$, we have

$w_{k}=\frac{\left(1 / \Delta x_{k}\right)}{w}, \quad w=\sum_{k=1}^{n}\left(1 / \Delta x_{k}\right)$

The weighted mean $S^{w}$ is expressed as

$S^{w}=\sum_{k=1}^{n} w_{k} \cdot S_{k}=\sum_{k=1}^{n} w_{k} \cdot \frac{y_{k}}{x_{k}}$ 
By error propagation, the accuracy of $S^{w}$, which is denoted as $\Delta S^{w}$, can be derived from Eq. (4).

$$
\Delta S^{w}=\sqrt{\sum_{k=1}^{n} w_{k}^{2} \cdot\left[\left(\frac{1}{x_{k}}\right)^{2} \cdot\left(\Delta y_{k}\right)^{2}+\left(\frac{-1}{x_{k}^{2}}\right)^{2} \cdot\left(\Delta x_{k}\right)^{2}\right]}
$$

With $S^{w}$ and $\Delta S^{w}$, the calibration factor $C$ and its accuracy $\Delta C$ are computed (assuming $C_{\text {apriori }}$ is errorless) as

$\left\{\begin{array}{l}C=S^{w} \cdot C_{\text {apriori }} \\ \Delta C=\Delta S^{w} \cdot C_{\text {apriori }}\end{array}\right.$

\section{DETERMINATING THE CALIBRATION FAC- TORS OF LCR-ET20 AND SG057}

\subsection{Calibration Factor of the LCR-ET20}

The preliminary calibration factor of the gravimeter LCR-ET20 is given by the manufacturer based on the mechanical calibration factor of each circle of the reading wheel. It is given as of $0.15488 \times 10^{-8} \mathrm{~m} \mathrm{~s}^{-2}$. Based on the mechanical calibration factor, the calibration factor of LCRET20 was determined at the Wuhan station as of (42.9185 \pm $0.0043) \times 10^{-8} \mathrm{~m} \mathrm{~s}^{-2} \mathrm{~V}^{-1}$. Because the mechanical calibration factor may have changed after several decades (Mao et al. 1989; Chen et al. 2003), the calibration factor of LCR-ET20 should be re-determined using other references. In this paper, the Wuhan ITGRVs from three main waves O1, K1 and M2 (Xu et al. 2000) were used to calculate the new calibration factor with the method given in section 2. The ITGRVs at the Wuhan station are determined based on the comprehensive analysis of the tidal gravity observations obtained from eight instruments, including four LCR, one SG and three geodynamical gravimeters (Xu et al. 2000).

The tidal gravity observations of LCR-ET20 at the Wuhan station that were used in the calculation are from August 20, 2008 to July 15, 2009. The tidal parameters of the main waves were obtained from the harmonic analysis by Eterna3.3, which is a standard software recommended by the International Center for Earth Tides (ICET) (Wenzel 1996). The observed amplitude factors and their accuracies for three main waves O1, K1 and M2 are given in Table 1.

Table 1. Observed and reference amplitude factors and their accuracies at Wuhan station.

\begin{tabular}{ccc}
\hline Main waves & $\begin{array}{c}\text { Observed amplitude } \\
\text { factors and accuracies } \\
\text { (LCR-ET20) }\end{array}$ & $\begin{array}{c}\text { Reference values } \\
\text { (ITGRVs) }\end{array}$ \\
\hline O1 & $1.17785 \pm 0.00187$ & $1.1780 \pm 0.0040$ \\
K1 & $1.14852 \pm 0.00156$ & $1.1522 \pm 0.0015$ \\
M2 & $1.17411 \pm 0.00045$ & $1.1751 \pm 0.0046$ \\
\hline
\end{tabular}

For comparison, the ITGRVs are also listed in this table. According to Eq. (4), the mean $S^{w}$ is 1.001176 suggesting that the change of the calibration factor is around $1 \%$. Therefore, the new calibration factor and its accuracy are $(42.9690 \pm 0.1139) \times 10^{-8} \mathrm{~m} \mathrm{~s}^{-2} \mathrm{~V}^{-1}$. It was found that the calibration factor has a very small change since LCR-ET20 was purchased in 1985 .

LCR-ET20 was then re-installed at Lhasa in November 2009 to calibrate SG057. By checking its reading wheel performance, we confirm that, the move from Wuhan to Lhasa did not change the calibration factor of the LCR-ET20. Therefore, the newly determined calibration factor of the LCR-ET20 can be regarded as a known value to be used for determining the calibration factor of SG057.

\subsection{Calibration Factor of the SG057}

The latitude, longitude and height of SG057 are $29.6450^{\circ} \mathrm{N}, 91.0350^{\circ} \mathrm{E}$ and $3632.30 \mathrm{~m}$, respectively. The data time span of SG057 is from December 8, 2009 to February 27, 2011 (447 days). The calibration factor of SG057 given by the manufacturer is the apriori calibration factor. The data recorded by LCR-ET20 at Lhasa has been calibrated by the newly determined calibration factor stated in section 3.1. The time span of the data is from November 27, 2009 to November 1, 2010 (321.4 days). The different data time spans are expected to have a small impact on the final calibration factor with regard to SG057 because the calibration is made in the frequency domain. The key point is to collect the longest records of data to produce the best harmonic analysis results. Again, Eterna3.3 is also used to estimate the tidal parameters. The observed amplitude factors and their accuracies from LCR-ET20 and SG057 for three main waves $\mathrm{O} 1, \mathrm{~K} 1$ and $\mathrm{M} 2$ are shown in Table 2. These amplitude factors are used to compute a new calibration factor of SG057.

With the result listed in Table 2, the calibration factor of SG057 is determined to be $(-77.7358 \pm 0.0409) \times 10^{-8}$ $\mathrm{m} \mathrm{s}^{-2} \mathrm{~V}^{-1}$. The estimated value $S^{w}$ is $0.977809 \pm 0.000515$. The relative accuracy is about $0.5 \%$. The absolute value of the calibration factor of SG057 is decreased from the apriori value of $79.5 \times 10^{-8}$ to $77.7358 \times 10^{-8} \mathrm{~m} \mathrm{~s}^{-2} \mathrm{~V}^{-1}$, and

Table 2. Observed amplitude factors and their accuracies of LCRET20 and SG057 for computing the new calibration factor of SG057.

\begin{tabular}{ccc}
\hline Main waves & $\begin{array}{c}\text { Observed amplitude } \\
\text { factors and accuracies } \\
\text { (LCR-ET20) }\end{array}$ & $\begin{array}{c}\text { Observed amplitude } \\
\text { factors and accuracies } \\
\text { (SG057) }\end{array}$ \\
\hline O1 & $1.17138 \pm 0.00294$ & $1.19707 \pm 0.00061$ \\
K1 & $1.13560 \pm 0.00206$ & $1.16947 \pm 0.00044$ \\
M2 & $1.17209 \pm 0.00050$ & $1.19891 \pm 0.00010$ \\
\hline
\end{tabular}


is about $2.2 \%$ smaller. The decrease should not be neglected because a typical relative accuracy of observed amplitude factor is better than 1\% for SG (Meurers 2012). This new calibration factor from the SG057 will be used for converting SG readings to gravity observations. This calibration factor will be submitted to the ICET for the Lhasa station.

\section{TIDAL AMPLITUDES AND PHASES AT LHASA WITH THE CALIBRATED SG057}

With the newly determined calibration factor, the gravity observations of SG057 were obtained and pre-processed using TSoft software (Van Camp and Vauterin 2005) to remove spikes, steps, gaps, and anomalous values caused by earthquakes and other disturbances. The purpose of the pre-processing is to prepare the data for the harmonic analysis. The sampling interval of the pre-processed data was 1 minute, but the data were resampled into a 1-hour sampling interval because the interested tidal gravity waves have periods longer than one hour. The tidal analysis result of SG057 is given in Table 3. In the analysis, the tidal potential catalogue developed by Tamura (1987) was used. Data filtering was also used to remove the long tidal gravity waves; and, the filter used in Eterna3.3 (name of the file: N60M60M2.NLF) has a cut-off frequency of $0.721499 \mathrm{cpd}$ (cycle per day). Therefore, only the tidal components with frequencies larger than $0.721499 \mathrm{cpd}$ were analyzed. In addition, the station air pressure effect was removed using a linear regression method, for which the atmosphere-gravity admittance is $(-3.63335 \pm 0.00762) \times 10^{-9} \mathrm{~m} \mathrm{~s}^{-2} \mathrm{hPa}^{-1}$. The standard deviation of the gravity residual is about $0.5 \times$ $10^{-9} \mathrm{~m} \mathrm{~s}^{-2}$

\section{THE GRAVIMETER DRIFT OF SG057}

To estimate the gravimeter drift of SG057, the synthesized gravity tide must be computed in advance. The synthesized gravity tide was computed using the tidal parameters listed in Table 3 and the theoretical amplitude factors for the long-period waves (the frequency band is from 0.0 to $0.721498 \mathrm{cpd}$ ) obtained from the Dehant-Defraigne-Wahr (DDW) earth tide model with a value of 1.1356 (Dehant et al. 1999). The phase leads for all long-period waves are set to 0 degree. The gravity tide generating program of TSoft was used to compute the synthesized gravity tide. Figure 1 shows the synthesized gravity tide and the gravity residual before and after the station air pressure correction was applied. In Fig. 1c, the gravity residual becomes smoother and smaller after the correction with air pressure. This suggests that the local atmosphere pressure has a large influence on the raw gravity observations and must be considered when processing the data from SG057. In addition, the gravity pole tide should be removed from the gravity residual. The gravity pole tide (Fig. 2a) was computed using the theoreti- cal amplitude factor of $\delta^{\text {th }}=1.16$ as recommended by ICET (Dehant et al. 1999); and, the Earth's rotation parameters (polar motion and UT1) were from International Earth Rotation and References Service System (IERS).

The gravity change caused by the uplift near SG057 has been observed by the FG5-112 absolute gravimeter (Zhang et al. 2001). The uplift causes gravity to decrease by $2 \times 10^{-8} \mathrm{~m} \mathrm{~s}^{-2} \mathrm{yr}^{-1}$ from 1993 to 1999 (Fig. 3a). Under the assumption that the gravity change rate at the Lhasa SG station is caused purely by the uplift, the drift rate of SG057 can be obtained from the gravity residual (Fig. 3b). Because an exponential drift did not occur at the beginning of the installation, the gravimeter drift of SG057 was assumed to be linear (Fig. 3b). With a linear fit to the gravity residual of SG057, the estimated gravimeter drift rate of SG057 is $6.8 \times$ $10^{-8} \mathrm{~m} \mathrm{~s}^{-2} \mathrm{yr}^{-1}$. This drift rate is very large compared to other SGs in the world. For example, Hwang et al. (2009) reported a drift rate of $0.2 \times 10^{-8} \mathrm{~m} \mathrm{~s}^{-2} \mathrm{yr}^{-1}$ for SG048 at Hsinchu. The exact causes of the gravity change rate given in Fig. $3 b$ will be a subject of future study.

When the gravimeter drift was removed, the gravity residual at Lhasa from December 2009 to September 2011 were computed and are shown in Fig. 4. Figure 4 shows that the peak-to-peak amplitude of the gravity residual is about $8 \times 10^{-8} \mathrm{~m} \mathrm{~s}^{-2}$, with a clear seasonal variation. The seasonal variation may be caused by the hydrological effect and will be investigated in future studies.

\section{CONCLUSIONS AND DISCUSSIONS}

In this paper, the calibration factor of SG057 was determined for the first time using the frequency-domain approach. The newly determined calibration factor is $(-77.7358 \pm 0.0409) \times 10^{-8} \mathrm{~m} \mathrm{~s}^{-2} \mathrm{~V}^{-1}$. Using the Wuhan ITGRVs, the newly determined calibration factor of SG057 will be consistent with the gravity observations at the $\mathrm{Wu}$ han station. The standard deviation of the gravity residual is about $0.5 \times 10^{-9} \mathrm{~m} \mathrm{~s}^{-2}$, suggesting that the noise level of SG057 is of the order of $10^{-9} \mathrm{~m} \mathrm{~s}^{-2}$. With the new calibration factor for SG057, the tidal parameters at the Lhasa station were computed (section 4). The synthesized gravity tide can provide accurate tidal gravity corrections for future gravity observations (from superconducting, absolute or relative gravimeters) in the Tibetan area.

With the gravimeter drift removed, the two-year gravity residual from SG057 shows a clear annual variation. More absolute gravity observations are needed to model the drift of SG057. The vertical displacement from continuous Global Positioning System (GPS) measurements can also be used to see the relationship between the gravity variation and the vertical displacement around SG057. The gravity variation caused by the uplift at the Lhasa station can be detected by SG057, but cannot be separated from the gravimeter drift without using other types of observations. 
Table 3. Results of tidal analyses from records of the calibrated SG057 at the Lhasa station.

\begin{tabular}{|c|c|c|c|c|c|}
\hline \multicolumn{2}{|c|}{ Frequency band } & \multirow[b]{2}{*}{$\begin{array}{c}\text { Tidal } \\
\text { waves }\end{array}$} & \multirow{2}{*}{$\begin{array}{l}\text { Theoretical amplitude } \\
\qquad\left[10^{-9} \mathrm{~m} \mathrm{~s}^{-2}\right]\end{array}$} & \multirow{2}{*}{$\begin{array}{l}\text { Amplitude factor } \\
\text { and its accuracy }\end{array}$} & \multirow{2}{*}{$\begin{array}{l}\text { Phase lead and its } \\
\text { accuracy [degree] }\end{array}$} \\
\hline $\begin{array}{c}\text { From } \\
\text { [cycle per day] }\end{array}$ & $\begin{array}{c}\text { To } \\
\text { [cycle per day] }\end{array}$ & & & & \\
\hline 0.721499 & 0.833113 & SGQ1 & 1.9721 & $1.15715 \pm 0.00635$ & $0.6037 \pm 0.3140$ \\
\hline 0.851181 & 0.859691 & 2Q1 & 6.7640 & $1.16603 \pm 0.00207$ & $0.6876 \pm 0.1016$ \\
\hline 0.860895 & 0.870024 & SGM1 & 8.1634 & $1.16960 \pm 0.00174$ & $0.4255 \pm 0.0852$ \\
\hline 0.887326 & 0.896130 & Q1 & 51.1178 & $1.17121 \pm 0.00029$ & $0.2541 \pm 0.0141$ \\
\hline 0.897806 & 0.906316 & RO1 & 9.7096 & $1.17388 \pm 0.00159$ & $0.4001 \pm 0.0776$ \\
\hline 0.921940 & 0.930450 & $\mathrm{O} 1$ & 266.9857 & $1.16760 \pm 0.00006$ & $-0.0177 \pm 0.0029$ \\
\hline 0.931963 & 0.940488 & TAU1 & 3.4822 & $1.15603 \pm 0.00435$ & $0.1410 \pm 0.2155$ \\
\hline 0.958085 & 0.966757 & NO1 & 20.9974 & $1.16244 \pm 0.00057$ & $-0.1104 \pm 0.0282$ \\
\hline 0.968564 & 0.974189 & CHI1 & 4.0158 & $1.16151 \pm 0.00401$ & $-0.3214 \pm 0.1978$ \\
\hline 0.989048 & 0.995144 & PI1 & 7.2651 & $1.14854 \pm 0.00234$ & $-0.9289 \pm 0.1167$ \\
\hline 0.996967 & 0.998029 & $\mathrm{P} 1$ & 124.2278 & $1.15579 \pm 0.00014$ & $-0.0147 \pm 0.0069$ \\
\hline 0.999852 & 1.000148 & S1 & 2.9377 & $1.53982 \pm 0.01572$ & $16.8227 \pm 0.6084$ \\
\hline 1.001824 & 1.003652 & $\mathrm{~K} 1$ & 375.4852 & $1.14166 \pm 0.00005$ & $0.0642 \pm 0.0022$ \\
\hline 1.005328 & 1.005624 & PSI1 & 2.9377 & $1.25073 \pm 0.00568$ & $-0.3220 \pm 0.2600$ \\
\hline 1.007594 & 1.013690 & PHI1 & 5.3469 & $1.17344 \pm 0.00307$ & $-0.2573 \pm 0.1503$ \\
\hline 1.028549 & 1.034468 & TET1 & 4.0151 & $1.16184 \pm 0.00389$ & $-0.1690 \pm 0.1916$ \\
\hline 1.036291 & 1.044801 & $\mathrm{~J} 1$ & 20.9966 & $1.16286 \pm 0.00076$ & $0.0336 \pm 0.0373$ \\
\hline 1.064840 & 1.071084 & $\mathrm{SO} 1$ & 3.4829 & $1.17787 \pm 0.00439$ & $-0.4062 \pm 0.2137$ \\
\hline 1.072582 & 1.080945 & OO1 & 11.4890 & $1.16080 \pm 0.00108$ & $0.0177 \pm 0.0531$ \\
\hline 1.099160 & 1.216398 & NU1 & 2.1997 & $1.16087 \pm 0.00532$ & $-0.0387 \pm 0.2627$ \\
\hline 1.719380 & 1.837970 & EPS2 & 4.1907 & $1.17959 \pm 0.00295$ & $-0.4028 \pm 0.1433$ \\
\hline 1.853919 & 1.862429 & $2 \mathrm{~N} 2$ & 14.3723 & $1.17759 \pm 0.00095$ & $-0.6030 \pm 0.0464$ \\
\hline 1.863633 & 1.872143 & MU2 & 17.3450 & $1.17674 \pm 0.00081$ & $-0.5122 \pm 0.0392$ \\
\hline 1.888386 & 1.896749 & $\mathrm{~N} 2$ & 108.6131 & $1.17147 \pm 0.00013$ & $-0.5806 \pm 0.0063$ \\
\hline 1.897953 & 1.906463 & NU2 & 20.6300 & $1.16947 \pm 0.00069$ & $-0.5995 \pm 0.0339$ \\
\hline 1.923765 & 1.942754 & M2 & 567.2884 & $1.16940 \pm 0.00002$ & $-0.4630 \pm 0.0012$ \\
\hline 1.958232 & 1.963709 & LAM2 & 4.1832 & $1.16885 \pm 0.00335$ & $-0.4328 \pm 0.1643$ \\
\hline 1.965826 & 1.976927 & $\mathrm{~L} 2$ & 16.0345 & $1.17034 \pm 0.00109$ & $-0.2953 \pm 0.0533$ \\
\hline 1.991786 & 1.998288 & $\mathrm{~T} 2$ & 15.4312 & $1.17141 \pm 0.00090$ & $-0.8316 \pm 0.0441$ \\
\hline 1.999705 & 2.000767 & S2 & 263.9324 & $1.16374 \pm 0.00006$ & $-0.6609 \pm 0.0043$ \\
\hline 2.002590 & 2.013690 & $\mathrm{~K} 2$ & 71.7503 & $1.16475 \pm 0.00018$ & $-0.3655 \pm 0.0090$ \\
\hline 2.031287 & 2.047391 & ETA2 & 4.0117 & $1.16438 \pm 0.00298$ & $0.0605 \pm 0.1465$ \\
\hline 2.067578 & 2.182844 & $2 \mathrm{~K} 2$ & 1.0507 & $1.15812 \pm 0.00773$ & $-0.2309 \pm 0.3822$ \\
\hline 2.753243 & 2.869714 & MN3 & 2.6583 & $1.08014 \pm 0.00275$ & $-0.0080 \pm 0.1459$ \\
\hline 2.892639 & 3.081255 & M3 & 9.6883 & $1.07900 \pm 0.00078$ & $0.0105 \pm 0.0416$ \\
\hline 3.791963 & 3.937898 & M4 & 0.1533 & $0.96286 \pm 0.02998$ & $-0.8779 \pm 1.7841$ \\
\hline
\end{tabular}




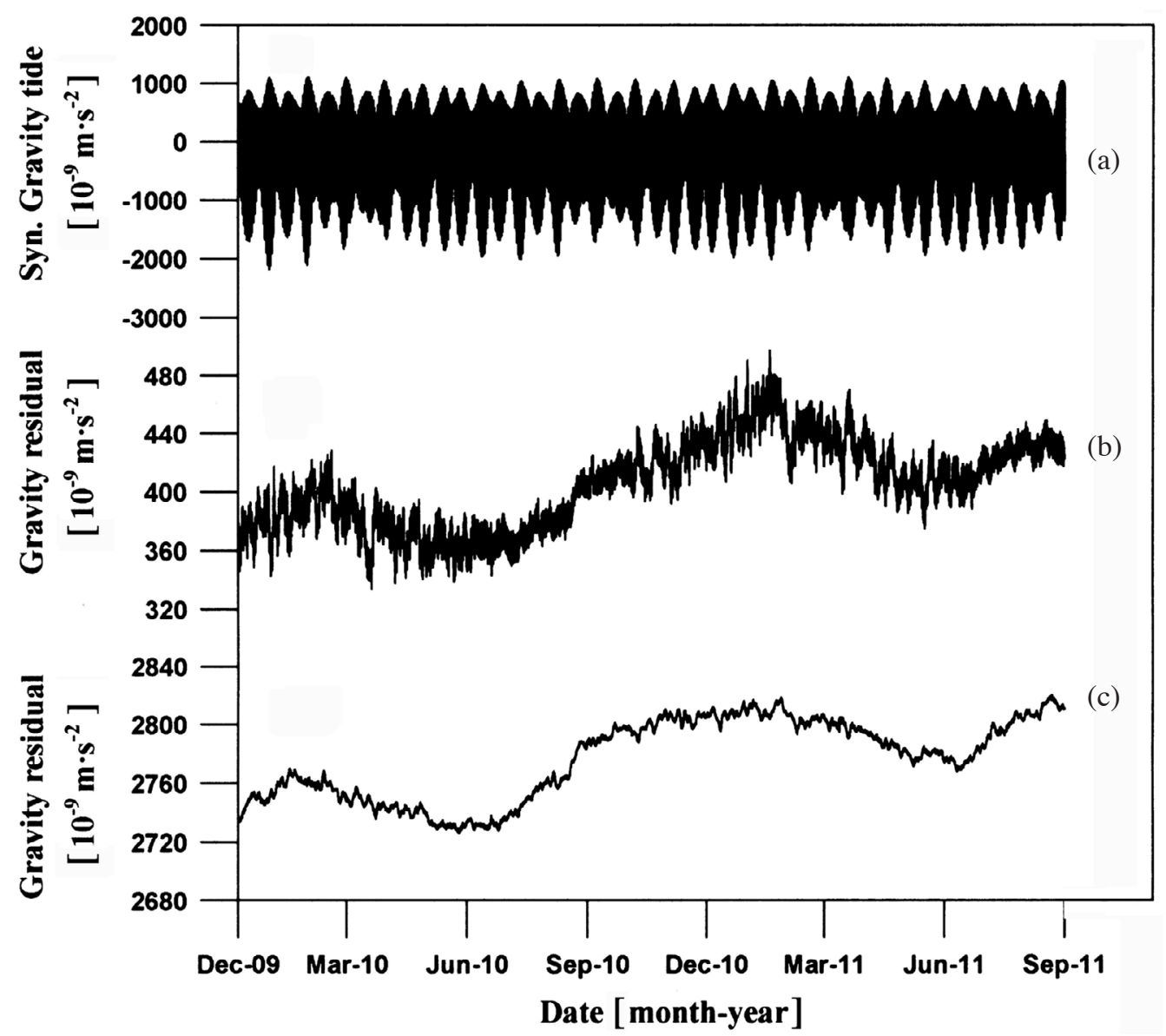

Fig. 1. Synthesized gravity tide (a) and gravity residual before (b) and after (c) station air pressure correction at the Lhasa station.

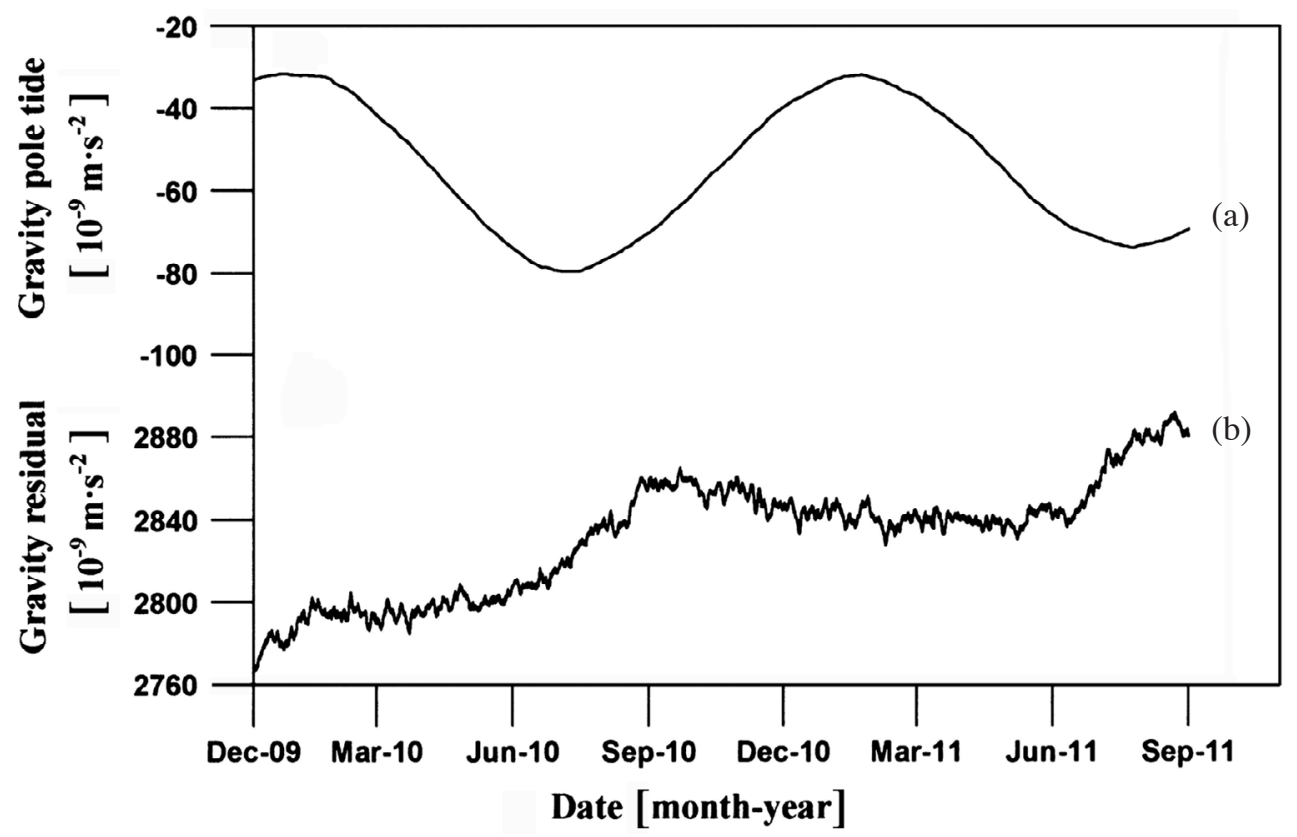

Fig. 2. Gravity pole tide (a) and gravity residual (b) with the pole tide removed from the Lhasa station. 


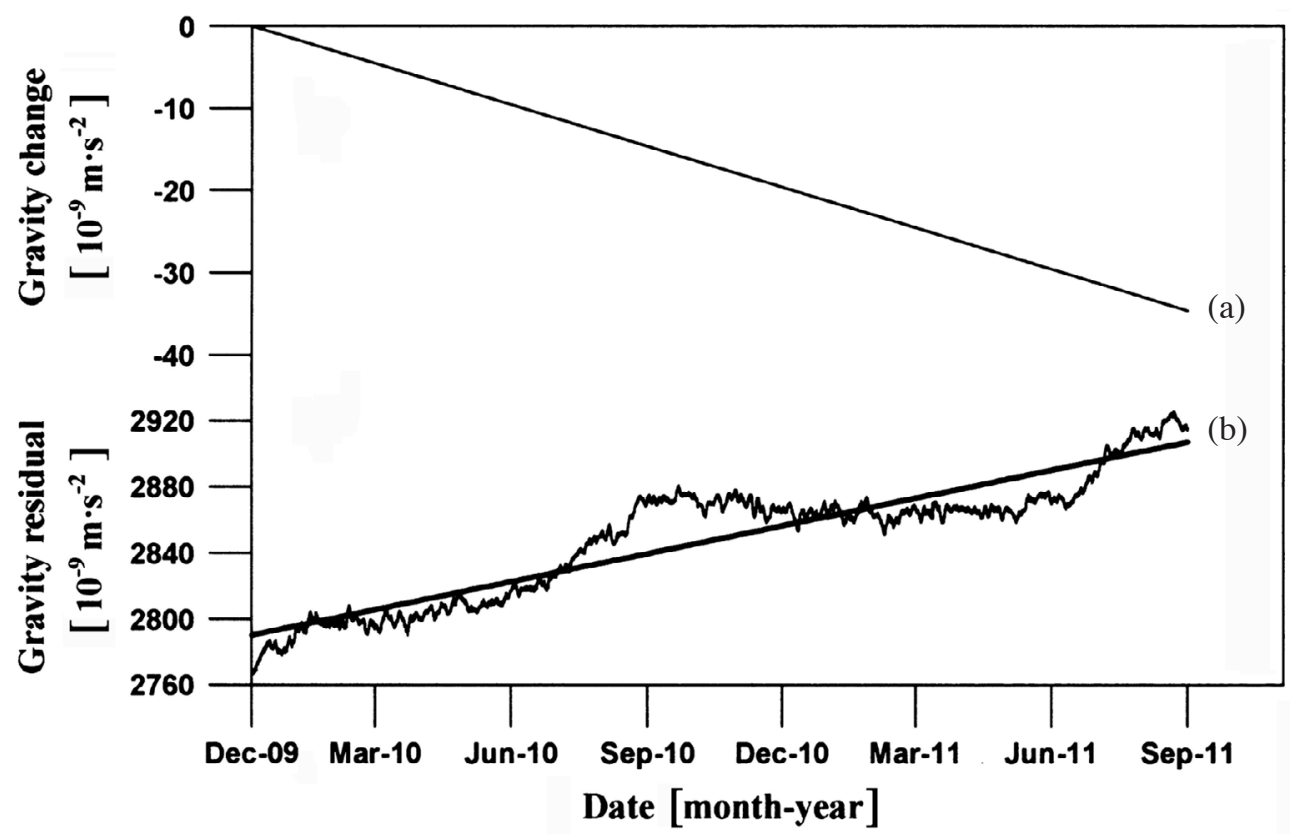

Fig. 3. Gravity change (a) caused by uplift observed by FG5-112 and (b) gravity residual with the uplift-induced rate removed. The fitted straight line in (b) is the gravimeter drift of SG057.

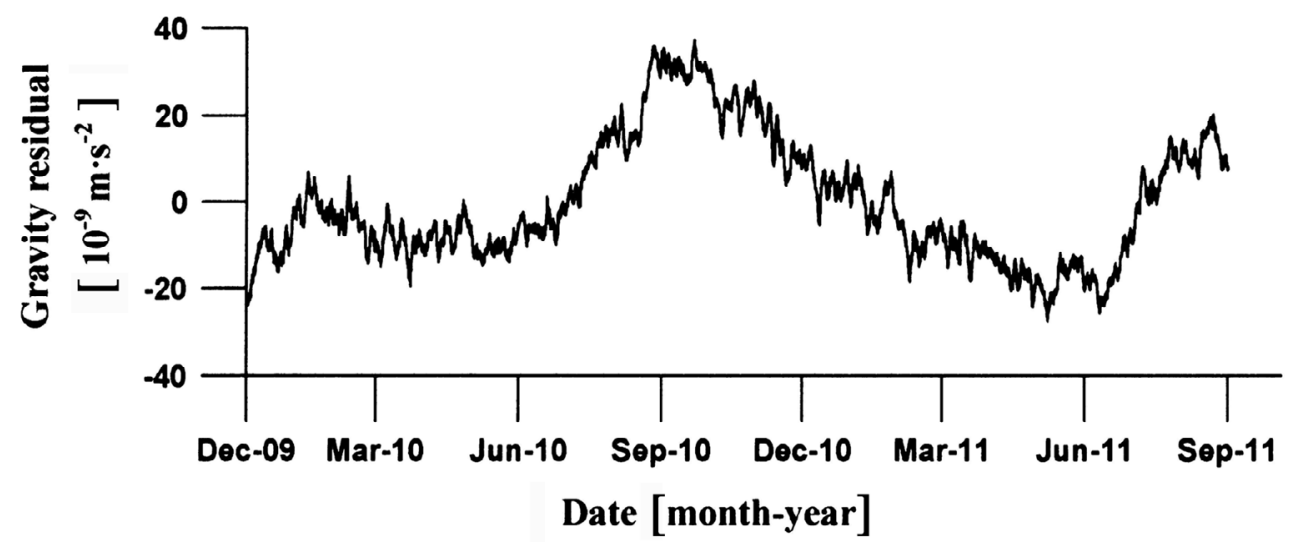

Fig. 4. Long-term behavior of the gravity variations at the Lhasa station.

Acknowledgements The study is supported by the Foundation for Innovative Research Groups of the National Natural Science Foundation of China (Grant No. 41021003), the National Natural Sciences Foundation of China (Grant No. 40904019, 41074053, 41004009) and the Foundation of the State Key Laboratory of Geodesy and Earth's Dynamics (Grant No. L10-09).

\section{REFERENCES}

Chen, X. D., H. P. Sun, M. Liu, and B. L. Zhou, 2003: Ac- curate determination of the scale factor of the LCRET20 gravimeter using observations recorded with the GWR-C032 superconducting gravimeter. Acta Geod. Cartogr. Sin., 32, 219-223. (in Chinese)

Crossley, D., J. Hinderer, and J.-P. Boy, 2005: Time variation of the European gravity field from superconducting gravimeters. Geophys. J. Int., 161, 257-264, doi: 10.1111/j.1365-246X.2005.02586.x. [Link]

Dehant, V., P. Defraigne, and J. M. Wahr, 1999: Tides for a convective Earth. J. Geophys. Res., 104, 1035-1058, doi: 10.1029/1998JB900051. [Link] 
Francis, O., 1997: Calibration of the C021 Superconducting gravimeter in Membach (Belgium) using 47 days of absolute gravity measurements. In: Segawa, J., H. Fujimoto, and S. Okubo (Eds.), Gravity, Geoid and Marine Geodesy, International Association of Geodesy Symposia, vol. 117, 212-219, International Symposium, Tokyo, Japan, September 30 - October 5, 1996.

Hinderer, J. and D. Crossley, 2004: Scientific achievements from the first phase (1997-2003) of the Global Geodynamics Project using a worldwide network of superconducting gravimeters. J. Geodyn., 38, 237-262, doi: 10.1016/j.jog.2004.07.019. [Link]

Hinderer, J., N. Florsch, J. Mäkinen, H. Legros, and J. E. Faller, 1991: On the calibration of a superconducting gravimeter using absolute gravity measurements. Geophys. J. Int., 106, 491-497, doi: 10.1111/j.1365-246X. 1991.tb03907.x. [Link]

Hwang, C., R. Kao, C. C. Cheng, J. F. Huang, C. W. Lee, and T. Sato, 2009: Results from parallel observations of superconducting and absolute gravimeters and GPS at the Hsinchu station of Global Geodynamics Project, Taiwan. J. Geophys. Res., 114, B07406, doi: 10. 1029/2008JB006195. [Link]

Kroner, C. and A. Weise, 2011: Sensitivity of superconducting gravimeters in central Europe on variations in regional river and drainage basins. J. Geodesy, $\mathbf{8 5}$, 651-659, doi: 10.1007/s00190-011-0471-1. [Link]

Lu, H., X. Wang, Z. An, X. Miao, R. Zhu, H. Ma, Z. Li, H. Tan, and X. Wang, 2004: Geomorphologic evidence of phased uplift of the northeastern Qinghai-Tibet plateau since 14 million years ago. Sci. China Earth Sci., 47, 822-833.

Mao, H. Q., H. T. Hsu, X. L. Song, and Z. B. Chen, 1989: East-west gravity tidal profile of China. Chin. J. Geophys., 32, 62-69. (in Chinese)

Meurers, B., 2002: Aspects of gravimeter calibration by time domain comparison of gravity records. BIM, 135, 10643-10650.

Meurers, B., 2012: Superconducting gravimeter calibration by colocated gravity observations: Results from GWR C025. Int.J. Geophys., 2012, 954271, doi: 10.11 55/2012/954271. [Link]
Riccardi, U., S. Rosat, and J. Hinderer, 2012: On the accuracy of the calibration of superconducting gravimeters using absolute and spring sensors: A critical comparison. Pure Appl. Geophys., 169, 1343-1356, doi: 10.10 07/s00024-011-0398-8. [Link]

Sun, H. P., X. D. Chen, H. T. Hsu, and Y. Wang, 2001: Accurate determination of calibration factor for tidal gravity observation of a GWR-superconducting gravimeter. Acta Seismol. Sin., 14, 692-700, doi: 10.1007/ BF02718080. [Link]

Tamura, Y., 1987: A harmonic development of the tidegenerating potential. BIM, 99, 6813-6855.

Van Camp, M. and O. Francis, 2007: Is the instrumental drift of superconducting gravimeters a linear or exponential function of time? J. Geodesy, 81, 337-344. doi: 10.1007/s00190-006-0110-4. [Link]

Van Camp, M. and P. Vauterin, 2005: Tsoft: Graphical and interactive software for the analysis of time series and Earth tides. Comput. Geosci., 31, 631-640, doi: 10.10 16/j.cageo.2004.11.015. [Link]

Wenzel, H. G., 1996: The nanogal software: Earth tide data processing package Eterna3.30. BIM, 124, 9425-9439.

Xing, L. L., W. K. Sun, H. Li, and G. L. Yang, 2011: Present-day crust thickness increasing beneath the QinghaiTibetan plateau by using geodetic data at Lhasa station. Acta Geod. Cartogr. Sin., 40, 41-44. (in Chinese)

Xu, H., H. Sun, J. Xu, and G. Tao, 2000: International tidal gravity reference values at Wuhan station. Sci. China Ser. D: Earth Sci., 43, 77-83, doi: 10.1007/BF02877 832. [Link]

Yi, H., C. Wang, Z. Shi, J. Lin, and L. Zhu, 2008: Early uplift history of the Tibetan Plateau: Records from paleocurrents and paleodrainage in the Hoh Xib Basin. Acta Geol.Sin., 82, 206-213.

Zhang, W., Y. Wang, H. Xu, C. Zhang, X. Hao, and M. Liu, 2001: Test of the uplift of Tibetan Plateau by FG5 absolute gravimeter at Lhasa station. Chin. Sci. Bull., 46, 256-258, doi: 10.1007/BF03187181. [Link]

Zhou, Z., Q. Yang, and K. Xia, 2007: Fossils of Quercus sect. Heterobalanus can help explain the uplift of the Himalayas. Chin. Sci. Bull., 52, 238-247, doi: 10.1007/ s11434-007-0005-7. [Link] 